\title{
The Research of Electric Energy Measurement Algorithm Based on S-Transform
}

\author{
Xiyang $\mathrm{Ou}^{1, *}$, Bei $\mathrm{He}^{2}$, Xiang $\mathrm{Du}^{1}$, Jin Zhang ${ }^{1}$, Ling Feng ${ }^{1}$, Chang $\mathrm{Ji}^{1}$ and Wanjun $\mathrm{Lei}^{3}$ \\ ${ }^{1}$ Sate Grid Chongqing Electric Power Research Institute, No. 80 Huangshan Road, Yubei, Chongqing, 401123, China \\ ${ }^{2}$ Sate Grid Chongqing Electric Power Company, No. 21 Zhongshan Road, Yuzhong, Chongqing, 400015, China \\ ${ }^{3}$ Xi'An Jiaotong University, No. 28 The West Road of Xianning, Beilin, Xi'an, 710049, China
}

\begin{abstract}
Considering the present electric energy measurement algorithm with the defect of low accuracy of measurement and unable to separate the fundamental wave and harmonic signal power when measurement the electric energy. This paper devises a fast and accurate electric energy measurement algorithm based on S-transform. Theoretical analysis shows that the S-transform can separate the signals into many components of different frequency, without the need for dividing the frequency band for many times to measure a harmonic component like wavelet packet algorithm, which greatly reduce the complexity of implementation and the subsequent error .In order to demonstrate the superiority of the method, this paper compares the results of the method based on S-transform algorithm with the measuring results of using the method of wavelet packet transform. Based on simulation analysis and experimental verification shows that, whether it is stationary or non-stationary signals signal, the measurement result of the method based on Stransform algorithm is greatly superior to the results which are obtained by wavelet packet transform.
\end{abstract}

Keywords-electric energy measurement algorithm; S-transform; harmonic decomposition; FFT

\section{INTRODUCTION}

As an important energy commodity, the accurate measurement of electrical energy related to the benefit of the generation side, supplier and the users. But with a large number of power electronic devices used in grid, resulting in a large number of grid harmonic signal, the electric energy metering device produce a great deal of measurement error, which will seriously affect the benefits of the generation side, supplier and the users in the grid and the rationality of the trade.

At present, the common electric energy measurement method in the electric system is Fast Fourier Transformation (FFT). Because the FFT integrates the signal on the total timedomain, FFT is unable to analysis the signal locally, the frequency spectrum of FFT has little time-domain information, only steady state signal can be analysis. So only the statistical property of the signal can be reflected by the Fourier spectrum. But for the certain frequency in the Fourier spectrum, the time of it begin and the time of it end can not be gained in the Fourier spectrum. So the FFT is not fit for non-stationary signal.

In recent years, the method of wavelet packet transform has been used in electric energy measurement. Compared with the FFT, the method of wavelet packet has the ability of localization analysis, it is very fit for detecting the singular signal and the unsteady state signal. The method of wavelet packet transform binary classify the low-frequency band and the high-frequency band. The bandwidth of the frequency band is smaller, the frequency components is more single, but excessive allocation band will intensify the complexity of the algorithmic and increase the algorithm error[1,2].

S-transform is a signal processing method came up with by R. G. Stockwell in 1996[3], equivalently the short-time Fourier transform with the normalized gaussian window or the wavelet transform with phase correction. S-transform is very fit for analyzing the singular signal and the unsteady state signal with its excellent time-frequency resolution. Besides, compared with the continuous wavelet transform, the S-transform is more precise and has better resolution especially in high frequency band. This paper puts forward a electric energy measurement algorithm based on S-transform decomposition and reconstruction, simulation analysis shows that the algorithm performance is excellent

\section{ELECTRIC ENERGy MEASUREMENT AlgORITHM BASED ON S-TRANSFORM}

S-transform has the property of lossless inversion . the signal can be translated into a matrix with the information of frequency and time by the theory of S-transform algorithm. Then the matrixes containing the information of only one frequency component can be gained by applying the linear separation on the matrix containing the information of the fundamental component and the harmonic components change over time. Then the time-domain information of the fundamental component and the harmonic components can be gained by applying inverse S-transform on the matrix. The energy of fundamental wave and harmonic wave can be gained by the formula $\sum u(k \Delta t) i(\Delta t) \Delta t$

\section{A. S-transform Frequency Spectrum Decomposition and Time-domain Signal Reconstruction}

This paper draw out the S-transform from the short time Fourier transform(STFT). The definition of STFT of Signal has been given as (1) :

$$
\operatorname{STFT}(t, f)=\int_{-\infty}^{+\infty} h(\tau) g(t-\tau) e^{-j 2 \pi f \tau} d \tau
$$

The S-transform definition formula can be gained by replacing the STFT rectangular function window with Gaussion window. The Gaussion window is defined as (2) : 


$$
g(t)=\frac{|f|}{\sqrt{2 \pi}} e^{-\frac{f^{2} t^{2}}{2}}
$$

So, the S-transform ${ }^{[4]}$ can be defined as (3) :

$$
S(t, f)=\int_{-\infty}^{+\infty} h(\tau) \frac{|f|}{\sqrt{2 \pi}} e^{-\frac{f^{2}(t-\tau)^{2}}{2}} e^{-j 2 \pi f \tau} d \tau
$$

The result matrix gained by S-transform: any row of the matrix shows the situation of amplitude and phase position change over time of a certain frequency, the frequency range from direct current to Nyquist frequency, any column of the matrix shows the amplitude and phase position of each frequency component at a certain time. So separating each frequency component can be realized by extracting the row element which represent each frequency component and constituting a new matrix which is the same size of the matrix that aforementioned and applying S-transform on it.

Known from the analysis above: S-transform and the converse transform is very important in metering the energy of each frequency individually.

Known as (4) :

$$
\int_{-\infty}^{+\infty} g(t-\tau) d t=\int_{-\infty}^{+\infty} \frac{|f|}{\sqrt{2 \pi}} e^{-\frac{f^{2}(t-\tau)^{2}}{2}} d t=1
$$

Time integration of the S-transform as (5) :

$$
\begin{aligned}
& \int_{-\infty}^{+\infty} S(t, f) d t \\
& =\int_{-\infty}^{+\infty}\left[\int_{-\infty}^{+\infty} h(\tau) \frac{|f|}{\sqrt{2 \pi}} e^{-\frac{f^{2}(t-\tau)^{2}}{2}} e^{-j 2 \pi f \tau} d \tau\right] d t \\
& =\int_{-\infty}^{+\infty}\left[\int_{-\infty}^{+\infty} h(\tau) \frac{|f|}{\sqrt{2 \pi}} e^{-\frac{f^{2}(t-\tau)^{2}}{2}} e^{-j 2 \pi f \tau} d t\right] d \tau \\
& =\int_{-\infty}^{+\infty} h(\tau) e^{-j 2 \pi f \tau}\left[\int_{-\infty}^{+\infty} \frac{|f|}{\sqrt{2 \pi}} e^{-\frac{f^{2}(t-\tau)^{2}}{2}} d t\right] d \tau \\
& =\int_{-\infty}^{+\infty} h(\tau) e^{-j 2 \pi f \tau} d \tau=H(f)
\end{aligned}
$$

So, Time integration of the S-transform is the Fourier spectrum of the signal ${ }^{[4]}$ as (6) :

$$
\begin{aligned}
& h(\tau)=\int_{-\infty}^{+\infty} H(f) e^{j 2 \pi f \tau} d \tau \\
& =\int_{-\infty}^{+\infty}\left[\int_{-\infty}^{+\infty} S(t, f) d t\right] e^{j 2 \pi f \tau} d \tau
\end{aligned}
$$

In conclusion, S-transform and inverse S-transform can realize the separation of the matrixes representing each frequency components and the reconstruction of the timedomain signal representing each frequency components.

\section{B. Discrete S-transform and Its Converse Transform}

In order to apply the electric energy measurement algorithm in computer system, the S-transform and its convers transform need to be discretized as (7):

$$
\begin{aligned}
S(t, f) & =\int_{-\infty}^{+\infty} x(\tau) m(t-\tau) e^{-j 2 \pi f \tau} d \tau \\
= & \int_{-\infty}^{+\infty} x(\tau) e^{-j 2 \pi f \tau} m(t-\tau) d \tau \\
= & {\left[x(t) e^{-j 2 \pi f t}\right] * m(t) } \\
= & F^{-1}[X(v+f)] * g(t)
\end{aligned}
$$

If the sampling time is $\Delta T$, the sampling frequency is $f_{s}=1 / \Delta T$, the number of sampling points is $N$, the frequency resolution is $\Delta F=f_{s} / N$. So $t=n \Delta T$, $f=m \Delta F, v=p \Delta F$.

So the discrete S-transform is (8) :

$$
\begin{aligned}
& S(n \Delta T, m \Delta F)= \\
& \left\{\begin{array}{l}
\sum_{p=0}^{N-1} X[p+m] e^{-\frac{2 \pi^{2} p^{2}}{m^{2}}} e^{j \frac{2 \pi p n}{N}}, m \neq 0 \\
\frac{1}{N} \sum_{k=0}^{N-1} x(k \Delta T), m=0
\end{array}\right.
\end{aligned}
$$

the discrete converse S-transform is (9) :

$$
x(k \Delta T)=\frac{1}{N} \sum_{m=0}^{N-1}\left\{\sum_{n=0}^{N-1} S(n \Delta T, m \Delta F)\right\} e^{j \frac{2 \pi k m}{N}}
$$

\section{The Implement of Electric Energy Measurement Algorithm Based on S-transform}

The time-domain signal of every frequency components can be obtained by the S-transform and the reverse transform. If the harmonic voltage and harmonic current is and separately ,the active energy of every harmonic in time quantum $\mathrm{T}$ is:

$$
W_{n}=\int_{0}^{T} u_{n}(t) i_{n}(t) d t \stackrel{\text { discret }}{=} \sum_{k=0}^{N-1} u[k] i[k] \Delta T
$$


The algorithm process of the electric energy measurement algorithm base on S-transform is shown in Figure I .

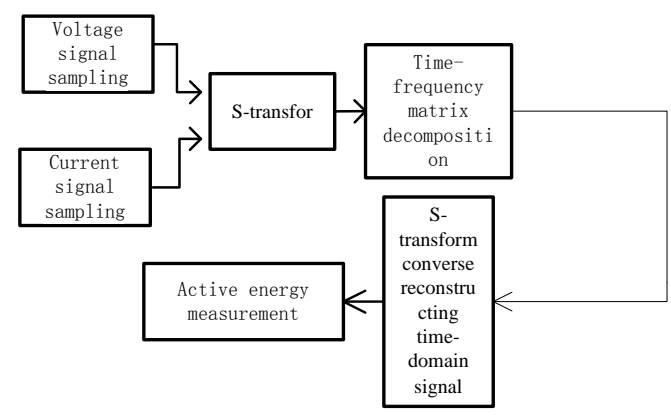

FIGURE I. THE ALGORITHM PROCESS OF THE ELECTRIC ENERGY MEASUREMENT ALGORITHM BASE ON S-TRANSFORM

\section{SiMULATED ANALYSIS AND EXPERIMENTAL VERIFICATION}

\section{A. Simulated Analysis}

The performance of electric energy measurement based on S-transform in the situation of steady state signal and unsteady state signal is simulated analyzed in MATLAB2012a. The parameter is set as follows: the frequency of fundamental wave $f_{0}=50 \mathrm{~Hz}$, the period of the fundamental wave $T=0.02 \mathrm{~s}$, sample frequency $f_{\mathrm{s}}=800 \mathrm{~Hz}$, simulation duration $f_{s}=800 \mathrm{~Hz}$. In order to verify that S-transform is more accurate than the wavelet packet transform, the simulate signal is constituted as in [5], the more accurate method can be found out by comparing the theoretical value, the result of wavelet packet and the result of S-transform.

1) Steady-state signal simulation

If the input signal and contains 3rd, 5th and 7th harmonic,

$$
\begin{gathered}
u(t)=100 \sin \left(2 \pi f_{0} t\right)+14 \sin \left(6 \pi f_{0} t\right)+ \\
12 \sin \left(10 \pi f_{0} t\right)+3 \sin \left(14 \pi f_{0} t\right) \\
0 \leq t \leq 40 T \\
i(t)=10 \sin \left(2 \pi f_{0} t\right)+4.5 \sin \left(6 \pi f_{0} t\right)+ \\
4 \sin \left(10 \pi f_{0} t\right)+2 \sin \left(14 \pi f_{0} t\right) \\
0 \leq t \leq 40 T
\end{gathered}
$$

The voltage waveform and the current waveform of every harmonic extracted from Steady-state signal by S-transform and its converse transform is shown in Figure II and Figure III (in order to show the waveform of the harmonic better, only 10T waveform is shown in Figure II and Figure III), the result of the active energy measure is shown in Table I.
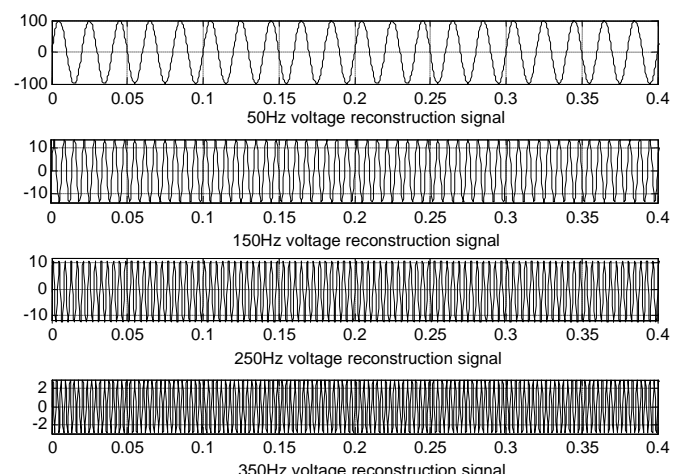

Time $\mathrm{t} / \mathrm{s}$

FIGURE II. THE RECONSTRUCTION OF STEADY-STATE VOLTAGE SIGNAL
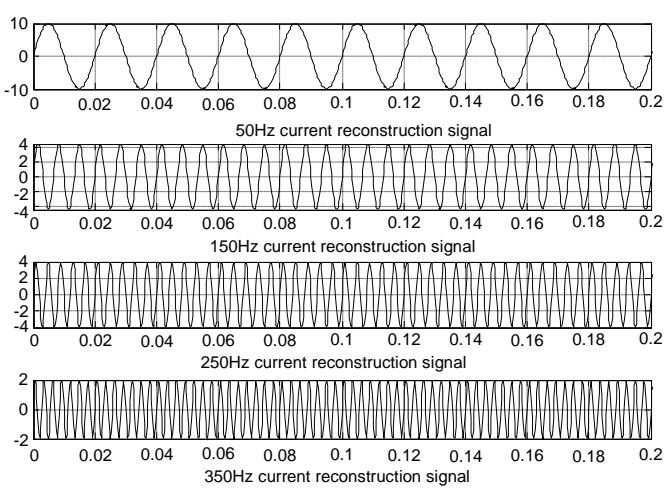

\begin{tabular}{|c|c|c|c|c|c|}
\hline \multirow{2}{*}{$\begin{array}{c}\text { freque } \\
\text { ncy } \\
(\mathrm{Hz})\end{array}$} & \multicolumn{3}{|c|}{ Active energy/J } & \multirow{2}{*}{$\begin{array}{c}\text { Error } \\
\text { of } \\
\text { wavele } \\
t \\
(\%)\end{array}$} & \multirow{2}{*}{$\begin{array}{c}\text { Error } \\
\text { of S } \\
(\%)\end{array}$} \\
\hline & $\begin{array}{l}\text { Truth- } \\
\text { value }\end{array}$ & wavelet & $S$ & & \\
\hline 50 & 300.0000 & 299.9785 & 300.0000 & $\begin{array}{c}- \\
0.0072\end{array}$ & 0.0000 \\
\hline 150 & 18.9000 & 18.8331 & 18.9000 & $\begin{array}{c}- \\
0.3540 \\
\end{array}$ & 0.0000 \\
\hline 250 & 14.4000 & 14.4643 & 14.4000 & 0.4467 & 0.0000 \\
\hline 350 & 1.8000 & 1.7999 & 1.8000 & $\begin{array}{c}- \\
0.0071\end{array}$ & 0.0000 \\
\hline Total & 335.1000 & 335.0758 & 335.1000 & $\begin{array}{c}- \\
0.0072\end{array}$ & 0.0000 \\
\hline
\end{tabular}

Times $t / s$

FIGURE III. THE RECONSTRUCTION OF STEADY-STATE CURRENT SIGNAL

TABLE I. THE SIMULATION DATA OF THE STEADY-STATE SIGNAL ACTIVE ENERGY

2) Unsteady-state signal simulation

The expression of the simulation signals are (13) and (14): 


$$
u(t)=\left\{\begin{array}{l}
100 \sin \left(2 \pi f_{0} t\right) \quad 0 \leq t<10 T \\
100 \sin \left(2 \pi f_{0} t\right)+ \\
20 \sin \left(6 \pi f_{0} t\right) \quad 10 T \leq t<20 T \\
100 \sin \left(2 \pi f_{0} t\right)+ \\
20 \sin \left(6 \pi f_{0} t\right)+ \\
17 \sin \left(10 \pi f_{0} t\right) \quad 20 T \leq t<25 T \\
100 \sin \left(2 \pi f_{0} t\right)+ \\
20 \sin \left(6 \pi f_{0} t\right)+ \\
17 \sin \left(10 \pi f_{0} t\right)+ \\
15 \sin \left(14 \pi f_{0} t\right) \quad 25 T \leq t<40 T
\end{array}\right.
$$$$
i(t)=\left\{\begin{array}{l}
10 \sin \left(2 \pi f_{0} t\right) \quad 0 \leq t<10 T \\
10 \sin \left(2 \pi f_{0} t\right)+ \\
2 \sin \left(6 \pi f_{0} t\right) \quad 10 T \leq t<20 T \\
10 \sin \left(2 \pi f_{0} t\right)+ \\
2 \sin \left(6 \pi f_{0} t\right)+ \\
1.8 \sin \left(10 \pi f_{0} t\right) \quad 20 T \leq t<25 T \\
10 \sin \left(2 \pi f_{0} t\right)+ \\
2 \sin \left(6 \pi f_{0} t\right)+ \\
1.8 \sin \left(10 \pi f_{0} t\right)+ \\
1.5 \sin \left(14 \pi f_{0} t\right) \quad 25 T \leq t<40 T
\end{array}\right.
$$
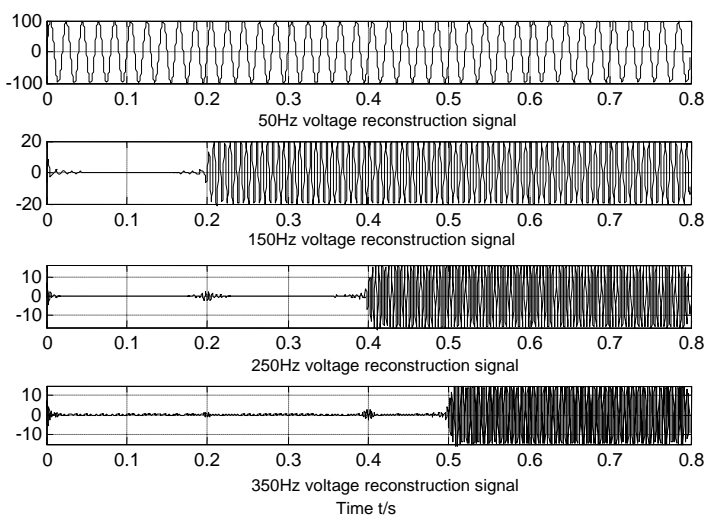

FIGURE IV. THE RECONSTRUCTION OF UNSTEADY-STATE VOLTAGE SIGNAL
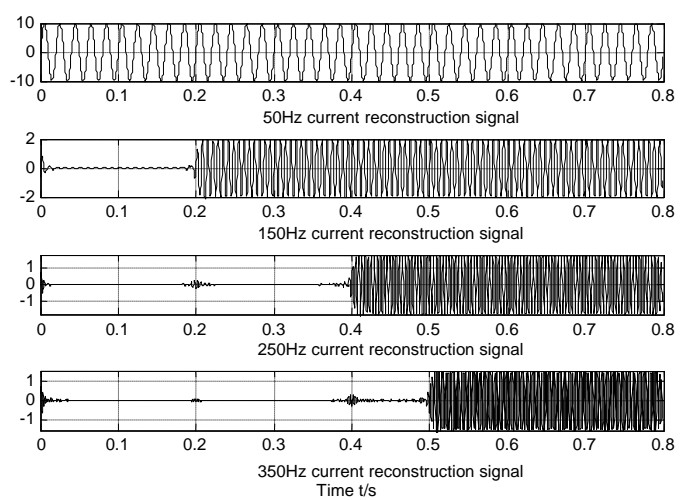

FIGURE V. THE RECONSTRUCTION OF UNSTEADY-STATE CURRENT SIGNAL

The voltage waveform and the current waveform of every harmonic extracted from unsteady-state signal by S-transform and its converse transform is shown in Figure IV and Figure V. The simulation result of S-transform applying on unsteadystate signal comparing with the result of wavelet packet and the theoretical value is shown in Table II.

TABLE II. THE SIMULATION DATA OF THE UNSTEADY STATE SIGNAL ACTIVE ENERGY

\begin{tabular}{|c|c|c|c|c|c|}
\hline \multirow{2}{*}{$\begin{array}{c}\text { Freq } \\
\text { uenc } \\
\mathrm{y} \\
(\mathrm{Hz})\end{array}$} & \multicolumn{3}{|c|}{ Active energy/J } & \multirow{2}{*}{$\begin{array}{c}\text { Error } \\
\text { of } \\
\text { wavele } \\
\text { t } \\
(\%)\end{array}$} & \multirow{2}{*}{$\begin{array}{c}\text { Error } \\
\text { of S } \\
(\%)\end{array}$} \\
\hline & $\begin{array}{l}\text { Truth- } \\
\text { value }\end{array}$ & wavelet & $S$ & & \\
\hline 50 & 300.0000 & 299.9894 & 300.0002 & $\begin{array}{c}- \\
0.0035\end{array}$ & 0.0001 \\
\hline 150 & 10.0000 & 9.9741 & 9.9902 & $\begin{array}{c}- \\
0.2590 \\
\end{array}$ & $\begin{array}{c}- \\
0.0980 \\
\end{array}$ \\
\hline 250 & 4.5900 & 4.6030 & 4.5839 & 0.2832 & $\begin{array}{c}- \\
0.1329\end{array}$ \\
\hline 350 & 2.2500 & 2.2597 & 2.2433 & 0.4311 & $\begin{array}{c}- \\
0.2978\end{array}$ \\
\hline $\begin{array}{c}\text { Tota } \\
\text { l }\end{array}$ & 316.8400 & 316.8262 & 316.8176 & 0.4518 & $\begin{array}{c}- \\
0.0071\end{array}$ \\
\hline
\end{tabular}

As known in chart 1, when measuring the active energy of the steady state signal, the result of S-transform is equal to the theoretical value if taking no account of the round-off error, the accuracy of S-transform is far above the wavelet packet transform. As known in Table II, as for the unsteady state signal, the accuracy of S-transform is above the wavelet packet transform too. The result of the simulation shows that, S-transform can extract each frequency components effectively, it is very fit for the active energy measurement of the steady state and the unsteady state signal.

\section{B. Experimental Verification}

In order to verify the effectiveness of the method mentioned above, a experimental platform of electrical energy measurement is constructed. The experimental platform is shown as Figure VI. 


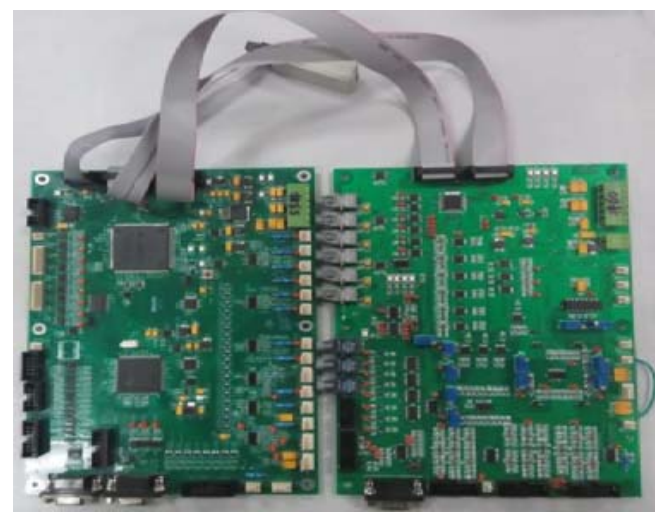

FIGURE VI. EXPERIMENTAL PLATFORM

The experimental platform is made up of, data processing circuit (using DSP chip TMS320F28335 of TI company), and D/A conversion circuit. The analog signal conditioning and sampling circuit turn the high voltage and large current into low voltage and small current, than a signal satisfying the A/D sampling chip's inputting requirement can be obtained after high frequency filtering, $\mathrm{A} / \mathrm{V}$ transformation and scaling transformation. This is the sampling work of the voltage and current, apply S-transform algorithm to realize electric energy measurement and is the core cell of the electric energy measurement system, it can output the value of the electric energy; D/A transform circuit turn the output of the data processing circuit into analog signal, and then show the result on the oscilloscope.

1) Steady state signal

If the steady state signals are (15) and (16):

$$
\begin{gathered}
u(t)=8 \sin \left(2 \pi f_{0} t\right)+4 \sin \left(6 \pi f_{0} t+30^{\circ}\right)+ \\
2 \sin \left(10 \pi f_{0} t+60^{\circ}\right) \quad 0 \leq t \leq 2 T \\
i(t)=6 \sin \left(2 \pi f_{0} t\right)+3 \sin \left(6 \pi f_{0} t\right)+ \\
1.5 \sin \left(10 \pi f_{0} t\right) \quad 0 \leq t \leq 2 T
\end{gathered}
$$

The waveform of the signal after decomposition and reconstruction is shown as Figure VII and Figure VIII, the measure result is shown in Table III.

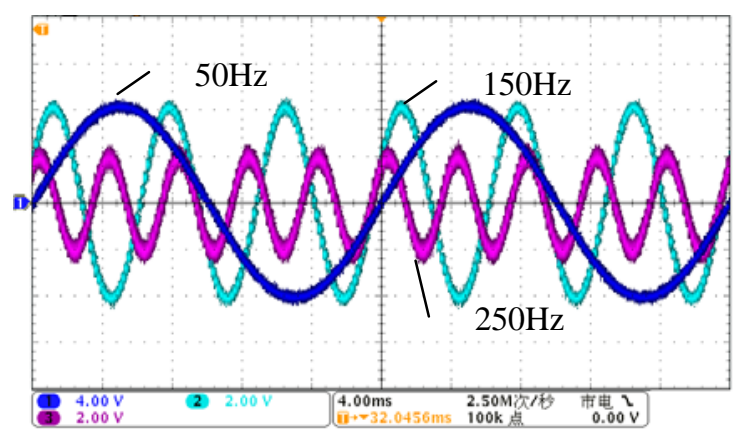

FIGURE VII. THE RECONSTRUCTION OF THE STEADY STATE VOLTAGE SIGNAL

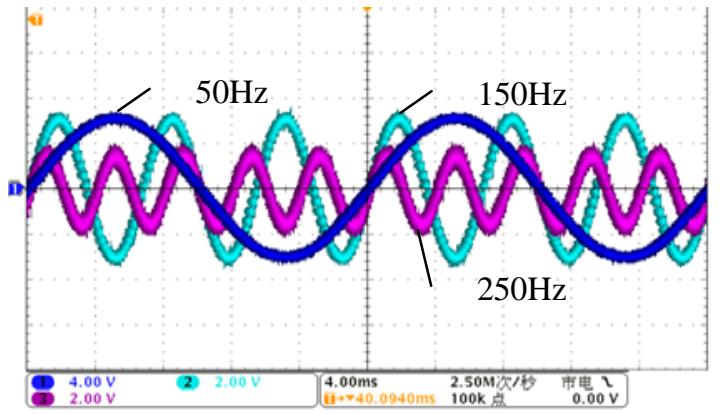

FIGURE VIII. THE RECONSTRUCTION OF STEADY STATE CURRENT SIGNAL

TABLE III. THE EXPERIMENTAL DATA OF THE STEADY-STATE SIGNAL ACTIVE ENERGY

\begin{tabular}{cccc}
\hline frequen & \multicolumn{2}{c}{ Active energy/J } & Error of S \\
\cline { 2 - 4 } cy & Truth-value & $\mathrm{S}$ & $\mathbf{( \% )}$ \\
\hline $\mathrm{Hz})$ & 0.9600 & 0.9600 & 0.0000 \\
150 & 0.2078 & 0.2078 & 0.0000 \\
250 & 0.0300 & 0.0300 & 0.0000 \\
\hline Total & 1.1978 & 1.1978 & 0.0000 \\
\hline
\end{tabular}

The experimental result shows that, S-transform can realize the decomposition and reconstruction of the steadystate signal, and it's high measuring accuracy can satisfy the requirement of practical application.

2) Unsteady-state signal

If the unsteady-state signals are (17) and (18):

$$
\begin{aligned}
& u(t)=\left\{\begin{array}{lc}
8 \sin \left(2 \pi f_{0} t\right) & 0 \leq t<2 T \\
8 \sin \left(2 \pi f_{0} t\right)+6 \sin \left(6 \pi f_{0} t+30^{\circ}\right) & 2 T \leq t<4 T
\end{array}\right. \\
& i(t)=\left\{\begin{array}{lr}
6 \sin \left(2 \pi f_{0} t\right) & 0 \leq t<2 T \\
6 \sin \left(2 \pi f_{0} t\right)+2 \sin \left(6 \pi f_{0} t\right) & 2 T \leq t<4 T
\end{array}\right.
\end{aligned}
$$

The waveform of the signal after decomposition and reconstruction is shown as Figure IX and Figure $\mathrm{X}$, the measure result is shown in Table IV .

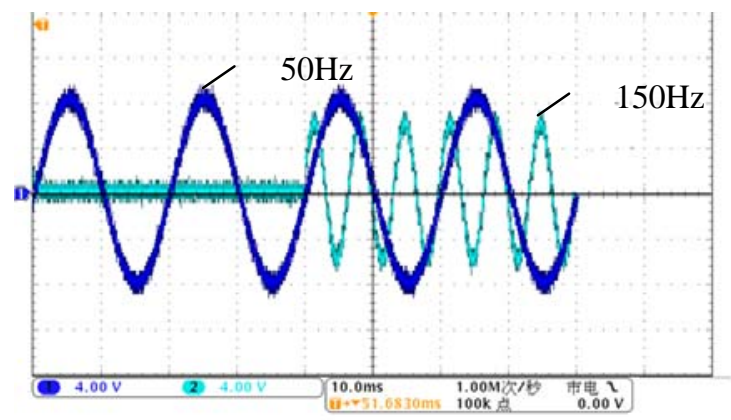

FIGURE IX. THE RECONSTRUCTION OF UNSTEADY STATE VOLTAGE SIGNAL 


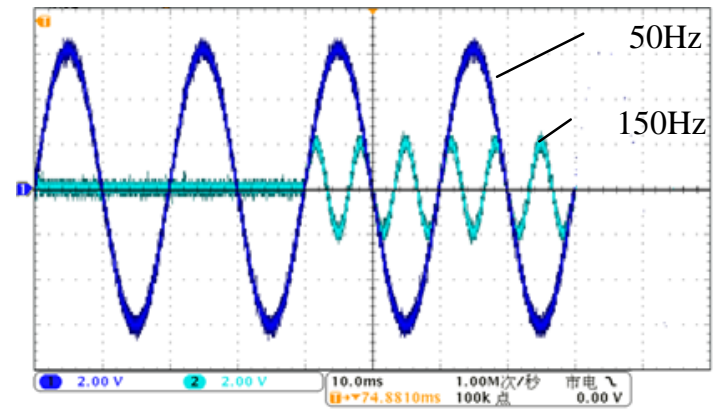

FIGURE X. THE RECONSTRUCTION OF UNSTEADY STATE CURRENT SIGNAL

TABLE IV. THE EXPERIMENTAL DATA OF THE UNSTEADY-STATE SIGNAL ACTIVE ENERGY

\begin{tabular}{cccc}
\hline frequen & \multicolumn{2}{c}{ Active energy/J } & Error of S \\
\cline { 2 - 4 } cy & Truth-value & $\mathrm{S}$ & $(\%)$ \\
\hline $\mathrm{Hz})$ & & & -0.0729 \\
\hline 50 & 0.9600 & 0.9593 & 0.0962 \\
\hline 150 & 0.2078 & 0.2080 & -0.0428 \\
\hline Total & 1.1678 & 1.1673 & \\
\hline
\end{tabular}

The experimental result shows that, S-transform can realize the decomposition and reconstruction of the steadystate signal, and it's high measuring accuracy can satisfy the requirement of practical application.

\section{CONCLUSION}

The electric energy measurement algorithm based on Stransform can extract the harmonic signal of different frequency accurately and reconstruct the time-domain signal. Then the energy of each harmonic component can be obtained. When measuring the electric energy, the method based on Stransform compared to wavelet packet can avoid a lot of problems, these problems such as very complex operating to obtain each harmonic component by applying binary average division to frequency band many times, or the closer frequency can not be separated thoroughly or interfere each other, but the S-transform can well deal it. The simulated analysis and the experimental verification show that the accuracy of the method based on S-transform is higher than the method based on wavelet packet transform both of steadystate signal and unsteady-state signal. So S-transform can satisfy the requirement of present electric energy measurement.

\section{REFERENCES}

[1] Mallat S. A wavelet tour of signal processing[M]. second edition (wavelet analysis \& its applications). San Diego: Academ ic Press, 1999.

[2] Julio Ban, Diego R I. A new method for measurementof harmonic groups in power systems using wavelet analysis in the IEC standard framework[J]. Electric Power Systems Research, 2006, 76(4): 200-208.

[3] Hongchun Shu. Application of signal processing in Power Engineering [M].Beijin: China Science Publishing, 2009, 363-368.

[4] R. G. Stockwell, L Mansinha and R P Lowe, "Localization of the complex spectrum: The S-transform,” IEEETrans. Signal Processing, vol. 44, no. 4, pp. 998-1001, April. 1996.
[5] Teng Zhaosheng, Luo Zhikun, Sun Chuanqi,Gao Yunpeng, Tang Qiu. Harmonic Energy Measurement Based on Wavelet Packet Decomposition and Reconstruction Algorithm [J]. Transactions of China Electrotechnical Society, 2010, 25(8):200-206 